\title{
Coronavirus disease (COVID-19) complicated by rhino-orbital-cerebral mucormycosis presenting with neurovascular thrombosis: a case report and review of literature
}

\author{
Vahid Reza Ostovan ${ }^{1}$ (D) Shirin Rezapanah ${ }^{1} \cdot$ Zahra Behzadi $^{1} \cdot$ Leila Hosseini $^{2} \cdot$ Reza Jahangiri $^{3}$. \\ Mohammad Hossein Anbardar ${ }^{4} \cdot$ Mahtab Rostami $^{1}$
}

Received: 13 December 2020 / Revised: 30 April 2021 / Accepted: 29 June 2021 / Published online: 3 August 2021

(c) Journal of NeuroVirology, Inc. 2021

\begin{abstract}
Among the ever-increasing literature of the coronavirus disease 2019 (COVID-19), there have been reports on several complications in association with severe acute respiratory syndrome-coronavirus-2 (SARS-CoV-2), such as secondary bacterial and fungal infections. We report a 61-year-old woman with a past history of diabetes mellitus who presented to our hospital suffering from COVID-19 infection. During the course of her hospitalization, the patient developed chemosis and proptosis in both eyes, ultimately leading to a diagnosis of invasive rhino-orbital-cerebral mucormycosis and cerebrovascular thrombosis. This study strengthens the possible association between the occurrence of COVID-19 and invasive mucormycosis infection, providing new impetus for further investigations to substantiate this correlation.
\end{abstract}

Keywords COVID-19 $\cdot$ SARS-CoV-2 $\cdot$ Mucormycosis $\cdot$ Thrombosis

\section{Introduction}

Acute invasive rhino-orbital-cerebral mucormycosis $(\mathrm{ROCM})$ is an infrequent, fatal infection that predominantly affects individuals with an immunocompromised state, a possible result of conditions, such as hematologic malignancies, diabetes mellitus, or organ transplantation (Hirabayashi et al. 2019). A novel coronavirus (severe acute respiratory syndrome-coronavirus-2 [SARSCoV-2]) emerged from China in December 2019. The disease has spread into other countries at a rapid rate and developed into a pandemic (Wang et al. 2020a). However, in regard to the relevant literature thus far,

Vahid Reza Ostovan ostovanv@gmail.com

1 Clinical Neurology Research Center, Shiraz University of Medical Sciences, Shiraz, Iran

2 Department of Internal Medicine, Shiraz University of Medical Sciences, Shiraz, Iran

3 Department of Otolaryngology, Shiraz University of Medical Sciences, Shiraz, Iran

4 Department of Pathology, Shiraz University of Medical Sciences, Shiraz, Iran fungal co-infection and secondary infection with SARSCoV-2 virus are relatively overlooked. In fact, there have been comparatively few reports published in the literature concerning the association of coronavirus disease 2019 (COVID-19) with fungal infection (Wang et al. 2020b; Chen et al. 2020a; Yang et al. 2020). Critically ill COVID19-infected individuals who need mechanical ventilation, or must stay in the hospital for 50 days or longer, or have an immunosuppressed health state are more vulnerable to contracting fungal co-infections (Yang et al. 2020). Several etiopathogenic mechanisms (e.g., a marked increment in some of the interleukins (IL) and cytokine storms, low levels of CD4 cells expressing interferongamma, and decrement in the total count of T-helper 1 and 2 cells) can contribute to the development of secondary invasive fungal infections in severely affected COVID19 patients (Chen et al. 2020b). Herein, we report a case of rhino-orbital-cerebral mucormycosis (ROCM) in association with COVID-19 infection and discuss the demographic, clinical, and prognostic characteristics of the aforementioned cases of sinonasal mucormycosis in association with COVID-19 in order to improve the diagnostic and treatment approaches in patients severely affected by SARS-CoV-2 and Rhizopus. 


\section{Case report}

A 61-year-old woman presented to our emergency department complaining of body pain, malaise, headache, hemianopia, and hemiparesis. She had a history of fatigue, generalized body pain, and progressive headache for 10 days preceding the admission. One day prior to the admission, sudden onset left-sided hemianopia and hemiparesis were added to her previous symptoms. Her past medical history (PMH) was notable for diabetes mellitus and hypertension. On arrival at the hospital, she was awake and oriented and her vital signs were normal with oxygen saturation $\left(\mathrm{SpO}_{2}\right)$ of $90 \%$ on room air. Neurological examination revealed left-sided homonymous hemianopia, left-sided hemiparesis with Medical Research Council (MRC) scoring of $0 / 5$ and $2 / 5$ in the left upper and lower extremities, respectively. Otherwise, the neurologic examination was unremarkable. Initial laboratory test results were as follows: white cell $\left(17.4 \times 10^{3} / \mu 1\right.$, with lymphocyte count $17 \%$, normal $\left.4-10 \times 10^{3} / \mu \mathrm{l}\right), \mathrm{C}$-reactive protein (CRP) (> $150 \mathrm{mg} / \mathrm{l}$, normal $0-6 \mathrm{mg} / \mathrm{l}$ ), erythrocyte sedimentation rate $(E S R)(138 \mathrm{~mm} / \mathrm{h}$, normal 0-30 mm/h), blood sugar (410, normal 80-140 mg/dl), hemoglobin A1c (12.8\%, normal <6\%), ferritin level $(4144 \mathrm{ng} / \mathrm{ml}$, normal 4-204 ng/ml), D-dimer (2700 ng/ml FEU, normal up to $500 \mathrm{ng} / \mathrm{ml} \mathrm{FEU}$ ), venous blood gas (VBG) (PH 7.56, normal 7.35-7.45; Hco3 $19.2 \mathrm{mmol} / \mathrm{l}$, normal 22-26 mmol/l), and negative serum ketone. Due to the flu-like symptoms and blood test results, real-time reverse-transcriptase polymerase chain reaction (RT-PCR) for SARS-CoV-2 using oropharyngeal/nasopharyngeal swabs (Roche, Penzberg, Germany) and lung high-resolution computed tomography scan (HRCT) were studied which were consistent with COVID-19 infection (Fig. 1A). Consequently, the patient was promptly treated with lopinavir/ritonavir 400/100 mg per oral (P.O.) twice daily, and her hyperglycemia was initially treated using lantus 10 units S.C (subcutaneous) qhs and sliding-scale protocol. The brain computed tomography (CT) showed hypodensities in the right frontal and parietooccipital junction regions suggestive of acute watershed infarct (Fig. 1B). On the 3rd day of admission, her level of consciousness was decreased with a Glasgow Coma Scale (GCS) of 8 out of 15; as a result, she was intubated. Due to the patient's condition worsening and her level of consciousness dropping and since her hyperglycemia was uncontrolled, the insulin intake protocol was changed from a subcutaneous protocol to a regular insulin IV infusion $1 \mathrm{unit} / \mathrm{h}$ regimen to maintain a blood glucose level of $150-180 \mathrm{mg} / \mathrm{dl}$. On the 5 th day of admission, she developed a GCS score of 6 out of 15 , with proptosis, chemosis, and negative doll's eye reflex in both eyes. Although the first brain CT scan showed no evidence of inflammation of the mucosal lining of the paranasal sinuses, the third one demonstrated a mucosal thickening of the paranasal sinuses, erosion of the right lamina papyracea with extraconal fat stranding, as well as hypodensities in the adjacent parts of the both frontal lobes (Fig. 1C-E). Furthermore, the brain CT venography was performed which showed filling defects in the bilateral cavernous sinuses and superior ophthalmic veins, predominantly on the right side, suggestive of cavernous sinus thrombosis (Fig. 1F, G). Consequently, unfractionated heparin 1000 units/h as a continuous IV infusion and amphotericin liposomal $10 \mathrm{mg} / \mathrm{kg}$ IV once daily (Cornely et al. 2019) were added to the patient's pervious treatment regimen. Subsequently, an otolaryngology consult was requested and the patient was scheduled for endoscopic sinus surgery. In the first step of the endoscopic sinus surgery, RT-PCR for SARS-CoV-2 was performed again from nasopharyngeal secretions using nasopharyngeal swab which was positive. Afterwards, ostium of all of the sinuses were opened, and necrotic tissues were debrided as much as possible. Pathologic examination of the necrotic tissue revealed broad and non-septated hyphae consistent with invasive mucormycosis (Fig. 1H). During the course of hospitalization, blood sugar was relatively controlled with regular insulin intravenous infusion and the patient had no evidence of metabolic acidosis. All of the vasculitis and hypercoagulable markers including protein $\mathrm{C}$, protein $\mathrm{S}$, factor $\mathrm{V}$ leiden, and antiphospholipid antibodies were within the normal limits. On the 11 th day of the admission, she became hypotensive and hemodynamically unstable; therefore, levophed was prescribed but the treatment was ineffective and the patient expired on the 12th day of the admission.

\section{Discussion}

Several factors, such as diabetes mellitus, neutropenia, systemic use of corticosteroid/immunosuppressive drugs, and hematologic malignancies can play a role as a predisposing factor for mucormycosis (Serris et al. 2019). Literature concerning the association of SARS-CoV-2 and fungal infections is scant with the prevalence of fungal co-infection, including invasive pulmonary aspergillosis and candidiasis in the setting of critically ill COVID-19 patients varying from 5 to $33.3 \%$ in these reports (Chen et al. 2020a; Yang et al. 2020; Alanio et al. 2020; van Arkel et al. 2020; Koehler et al. 2020). The combination of severe alveolo-interstitial inflammatory changes and damage, alteration of the immune response by SARS-CoV-2 virus including cytokine storms (marked elevation of both pro-inflammatory (IL-1, IL-2, IL-6, tumor necrosis alpha) and anti-inflammatory (IL-4, IL-10) 

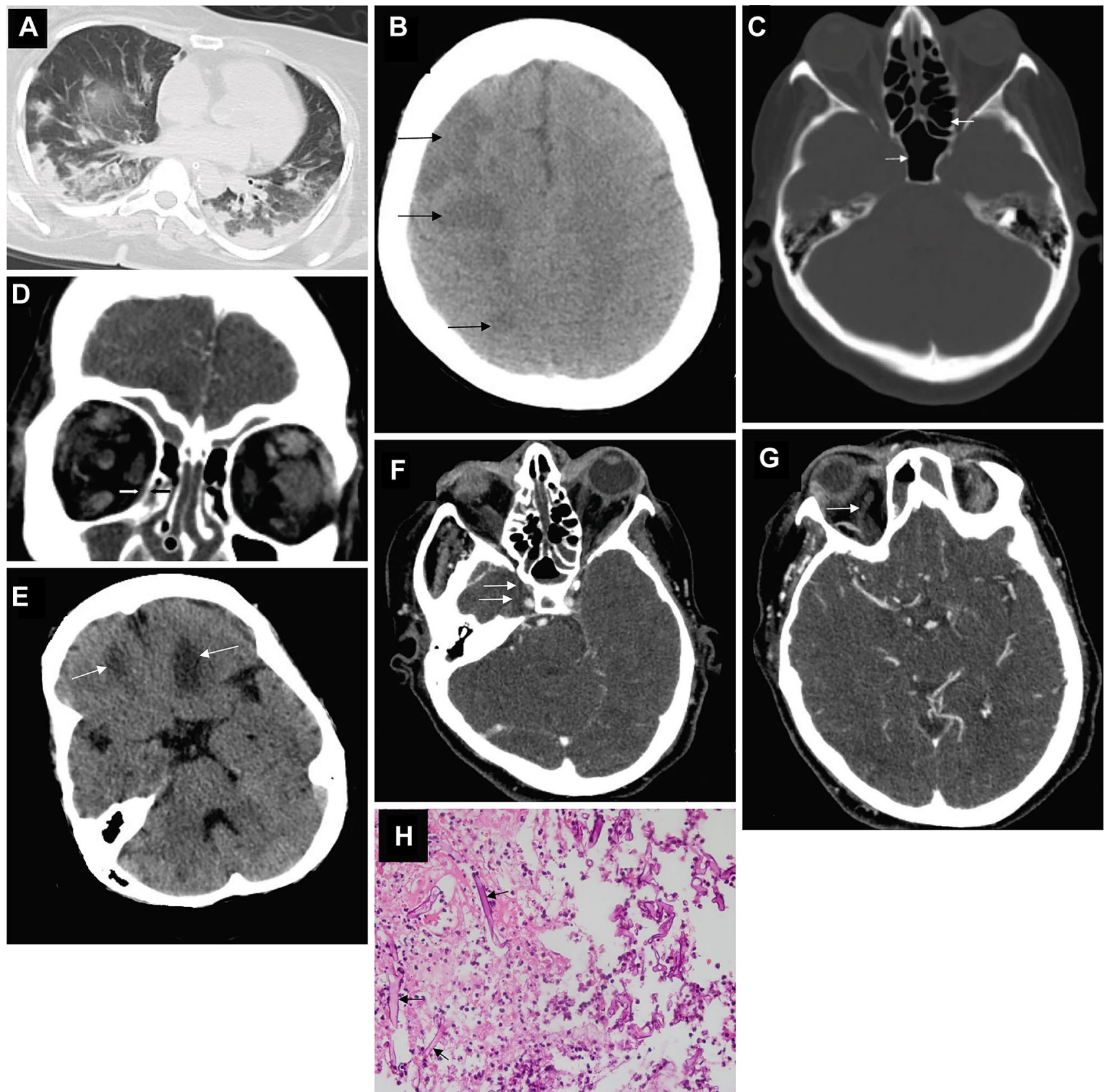

Fig. 1 A Lung high-resolution computed tomography (HRCT) shows several peripherally located patches of ground glass opacities in the lower lobes of both lungs highly suggestive of COVID-19. B Axial non-enhanced spiral brain CT scan shows hypodensities in the middle cerebral artery-posterior cerebral artery (MCA-PCA) and MCAanterior cerebral artery (MCA-ACA) junctions suggestive of acute watershed infarct (black arrows). C Axial non-enhanced spiral brain $\mathrm{CT}$ bone window on admission shows normal air-filled paranasal (ethmoidal and sphenoidal) sinuses (white arrows). D Paranasal sinuses CT scan (PNS CT) on the 5th day of admission shows mucosal thickening of the ethmoidal sinus with right lamina papyracea bone

cytokines), as well as reduced levels of both CD4 and CD8 T cells; hypoxia; the administration of immunomodulatory drugs such as systemic steroids, tocilizumab (anti-interleukin-6), and erosion (black thick arrow) associated with adjacent extraconal fat stranding (white thick arrow) suggestive of orbital involvement. E Axial non-enhanced spiral brain CT scan shows bilateral hypodensities in the base of both frontal lobes adjacent to the posterior ethmoidal sinuses (white arrows) indicative of intracranial extension of infection. F, G Brain CT venography (CTV) shows expansion of the right cavernous sinus (white arrows) as well as filling defects in the both cavernous sinuses and right superior ophthalmic vein (white arrow). $\mathbf{H}$ Microscopic section from sinonasal tissue shows necrotic background with many broad and non-septated mucormycosis hyphae (black arrows) (hematoxylin and eosin stain, $\times 400$ )

anakinra (recombinant interleukin-1Ra); and widespread use of broad-spectrum antibiotics, may result in an increased risk of secondary fungal infection in severe cases of COVID-19 


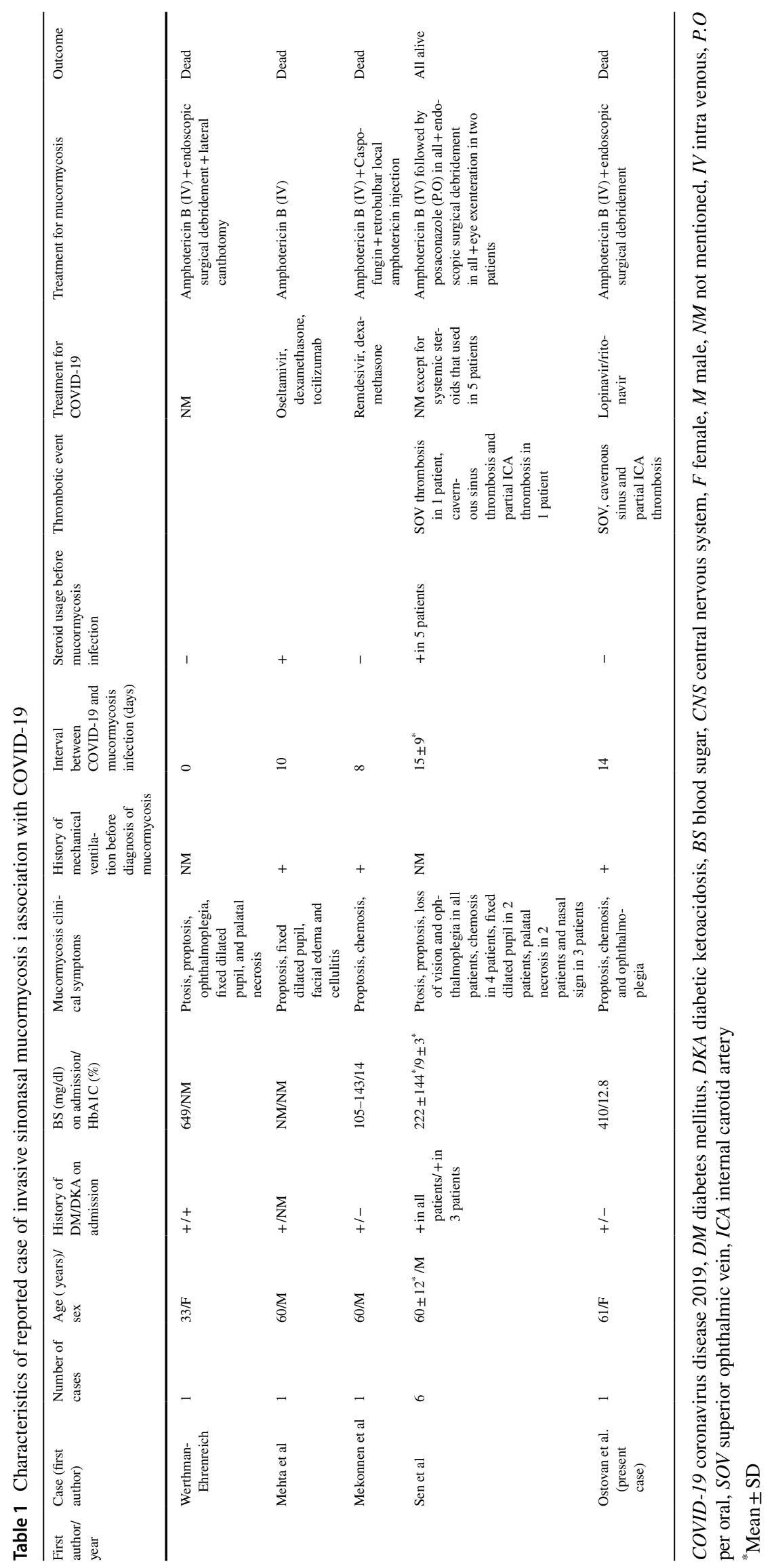


(Chen et al. 2020a, b; Koehler et al. 2020; Rawson et al. 2020; Armstrong-James et al. 2020). These factors contribute to the development of invasive pulmonary fungal diseases, but it is still unclear whether sinonasal tissue as an upper respiratory tract is likewise susceptible to fungal infections in critically ill COVID-19 patients.

To the best knowledge of the authors of the present study, thus far, only a few cases of invasive sinonasal mucormycosis in association with the COVID-19 infection have been reported in the literature, of which the demographic data, clinical characteristics, and outcomes are shown in Table 1 (Werthman-Ehrenreich 2020; Mehta and Pandey 2020; Mekonnen et al. 2021; Sen et al. 2021). Given the interval between the occurrence of COVID-19 and that of mucormycosis in the previously reported cases and in our study (only 2 out of 10 cases happened concurrently), it seems that SARS-CoV-2 has a provocative effect on the development of ROCM in patients. However, the existence of diabetes mellitus as the most common predisposing factor for mucormycosis in all of the patients makes it difficult to identify the relationship between COVID-19 and mucormycosis with certainty. As shown in Table 1, 6 out of 10 cases had a history of systemic steroids administration before the emergence of the mucormycosis-associated symptoms. As a result, systemic steroids can be considered as a potential risk factor for the development of invasive mucormycosis in the setting of SARS-CoV-2 infection, particularly in patients with uncontrolled hyperglycemia. Although prescribing systemic steroids is advised by UK RECOVERY Collaborative Group in critically ill COVID-19 patients (RECOVERY Collaborative Group et al. 2021), these case reports may raise concern about the safety of using systemic steroids in the context of severe COVID-19 infections and diabetes mellitus. Therefore, further studies and clinical trials are warranted to elaborate on the risk-benefit ratio of steroids in patients suffering from both diabetes mellitus and severe COVID-19.

Considering the rapid progression of ROCM in cases of severe COVID-19 infections in the previously reported cases as well as our own, it appears that mucormycosis has a more aggressive and relentless course when occurring in patients already suffering from a severe COVID-19 infection. However, more cases are required in order to obtain a more accurate assessment of the case fatality rate and the pathogenesis of mucormycosis in co-occurrence with COVID-19. Besides, a detailed review of the clinical characteristics of the previously reported cases and ours shows that a history of mechanical ventilation before the appearance of mucormycosis-associated symptoms is the worst prognostic factor and that all patients who needed mechanical ventilation ultimately expired.

ROCM is an invasive fungal infection with a 30-day mortality rate of $35 \%$ which increases to $66 \%$ in case of a 6-day delay in diagnosis and appropriate treatment initiation (Chamilos et al. 2008). Among the patients with invasive mucormycosis and COVID-19, 4 out of 10 were intubated or had decreased level of consciousness at the time of the mucormycosis diagnosis and they were not able to complain of mucormycosis-associated symptoms, which might have delayed a timely diagnosis and consequently increased the mortality rate. Additionally, if the patient is intubated, performing magnetic resonance imaging (MRI), as a gold standard imaging modality for the diagnosis of invasive sinonasal fungal disease, is not possible, which creates more difficulties for a correct diagnosis.

Systemic anti-fungal treatment and surgical debridement are the standard and accepted treatment for invasive rhino-orbital mucormycosis; however, in cases of reversible immunosuppression, good preoperative visual acuity, and low burden of orbital disease, local retrobulbar injection of liposomal amphotericin $B$ as an adjunctive treatment can effectively halt the progression of the fungal infection and reduce the exigency of performing highmorbidity procedures such as orbital exenteration (ColonAcevedo et al. 2015).

Although the exact role of COVID-19 in venous and arterial thrombosis is still being debated, 3 out of 10 patients displayed evidence of arterial or venous thrombosis of the central nervous system (Table 1), which might be due to the synergistic effects of SARS-CoV-2 and mucormycosis. SARS-CoV-2 can provoke a thrombotic cascade via various mechanisms, including the activation of the renin-angiotensin system, direct invasion of the endothelium, the activation of the complement system, and increasing the inflammatory biomarkers (IL- 6 and IL-1 $\beta$ ) (Tal et al. 2020).

As we live in the era of the COVID-19 pandemic and due to the high mortality and morbidity rates of ROCM in the context of COVID-19, regularly checking for the appearance of facial edema, facial cellulites, nasal or palatal necrosis, proptosis, chemosis, or ophthalmoplegia (particularly in immunocompromised individuals with a severe SARS$\mathrm{CoV}-2$ infection) is crucial in order to improve outcomes and reduce morbidity and mortality.

As a major limitation, the patient in our study had diabetes mellitus as a main predisposing factor of mucormycosis; therefore, we can't identify the causal relation between COVID-19 and mucormycosis with certainty. Moreover, due to the patient intubation early in the hospital course, magnetic resonance imaging (MRI) could not be performed to measure the severity of encephalitis with accuracy.

In conclusion, this case report implies a possible association between COVID-19 and invasive sinonasal fungal diseases. Many more cases and studies must yet be published for this correlation to be substantiated. 
Author contribution Material preparation and data collection were performed by VRO, SR, ZB, LH, RJ, MHA, and MR. The first draft of the manuscript was written by VRO, SR, ZB, LH, RJ, MHA, and MR and reviewed critically by VRO. All authors read and approved the final manuscript.

\section{Declarations}

Ethics approval and consent to participate The protocol of this study was approved by the ethics committee of Shiraz University of Medical Sciences (Approval No \# IR.SUMS.MED.REC.1400.104). All procedures followed were in accordance with the ethical standards of the responsible committee on human experimentation (institutional and national) and with the ethical standards laid down in the 1964 Declaration of Helsinki and its later amendments. Written informed consent was obtained from the patient's next of kin for the publication of her anonymized data in this case report.

Conflict of interest The authors declare no competing interests.

\section{References}

Alanio A, Dellière S, Fodil S, Bretagne S, Mégarbane B (2020) Prevalence of putative invasive pulmonary aspergillosis in critically ill patients with COVID-19. Lancet Respir Med 8:e48-e49

Armstrong-James D, Youngs J, Bicanic T, Abdolrasouli A, Denning DW, Johnson E, Mehra V, Pagliuca T, Patel B, Rhodes J, Schelenz S, Shah A, van de Veerdonk FL, Verweij PE, White PL, Fisher MC (2020) Confronting and mitigating the risk of COVID-19 associated pulmonary aspergillosis. Eur Respir J 56:2002554

Chamilos G, Lewis RE, Kontoyiannis DP (2008) Delaying amphotericin b-based frontline therapy significantly increases mortality among patients with hematologic malignancy who have zygomycosis. Clin Infect Dis 47:503-509

Chen N, Zhou M, Dong X, Qu J, Gong F, Han Y, Qiu Y, Wang J, Liu Y, Wei Y, Xia J, Yu T, Zhang X, Zhang L (2020a) Epidemiological and clinical characteristics of 99 cases of 2019 novel coronavirus pneumonia in Wuhan, China: a descriptive study. Lancet 395:507-513

Chen G, Wu D, Guo W, Cao Y, Huang D, Wang H, Wang T, Zhang X, Chen H, Yu H, Zhang X, Zhang M, Wu S, Song J, Chen T, Han M, Li S, Luo X, Zhao J, Ning Q (2020b) Clinical and immunologic features in severe and moderate coronavirus disease 2019. J Clin Invest 130:2620-2629

Colon-Acevedo B, Kumar J, Richard MJ, Woodward JA (2015) The role of adjunctive therapies in the management of invasive sinoorbital infection. Ophthal Plast Reconstr Surg 31:401-405

Cornely OA, Alastruey-Izquierdo A, Arenz D, Chen SCA, Dannaoui E, Hochhegger B, Hoenigl M, Jensen HE, Lagrou K, Lewis RE, Mellinghoff SC, Mer M, Pana ZD, Seidel D, Sheppard DC, Wahba R, Akova M, Alanio A, Al-Hatmi AMS, Arikan-Akdagli S, Badali H, Ben-Ami R, Bonifaz A, Bretagne S, Castagnola E, Chayakulkeeree M, Colombo AL, Corzo-León DE, Drgona L, Groll AH, Guinea J, Heussel CP, Ibrahim AS, Kanj SS, Klimko N, Lackner M, Lamoth F, Lanternier F, Lass-Floerl C, Lee DG, Lehrnbecher T, Lmimouni BE, Mares M, Maschmeyer G, Meis JF, Meletiadis J, Morrissey CO, Nucci M, Oladele R, Pagano L, Pasqualotto A, Patel A, Racil Z, Richardson M, Roilides E, Ruhnke M, Seyedmousavi S, Sidharthan N, Singh N, Sinko J, Skiada A, Slavin M, Soman R, Spellberg B, Steinbach W, Tan BH, Ullmann AJ, Vehreschild JJ, Vehreschild MJGT, Walsh TJ, White PL, Wiederhold NP, Zaoutis T, Chakrabarti A, for the Mucormycosis ECMM MSG Global Guideline Writing Group
(2019) Global guideline for the diagnosis and management of mucormycosis: an initiative of the European Confederation of Medical Mycology in cooperation with the Mycoses Study Group Education and Research Consortium. Lancet Infect Dis 19:e405-e421

Hirabayashi KE, Idowu OO, Kalin-Hajdu E, Oldenburg CE, Brodie FL, Kersten RC, Vagefi MR (2019) Invasive fungal sinusitis: risk factors for visual acuity outcomes and mortality. Ophthalmic Plast Reconstr Surg 35:535-542

Koehler P, Cornely OA, Bottiger BW, Dusse F, Eichenauer DA, Fuchs F, Hallek M, Jung N, Klein F, Persigehl T, Rybniker J, Kochanek M, Böll B, Shimabukuro-Vornhagen A (2020) COVID-19 associated pulmonary aspergillosis. Mycoses 63:528-534

Mehta S, Pandey A (2020) Rhino-orbital mucormycosis associated with COVID-19. Cureus 12:e10726. https://doi.org/10.7759/ cureus. 10726

Mekonnen ZK, Ashraf DC, Jankowski T, Grob SR, Vagefi MR, Kersten RC, Simko JP, Winn BJ (2021) Acute invasive rhino-orbital mucormycosis in a patient with COVID-19-associated acute respiratory distress syndrome. Ophthalmic Plast Reconstr Surg 37:e40-e80

Rawson TM, Moore LSP, Zhu N, Ranganathan N, Skolimowska K, Gilchrist M, Satta G, Cooke G, Holmes A (2020) Bacterial and fungal coinfection in individuals with coronavirus: a rapid review to support covid-19 antimicrobial prescribing. Clin Infect Dis 71:2459-2468. https://doi.org/10.1093/cid/ciaa530

RECOVERY Collaborative Group, Horby P, Lim WS, Emberson JR, Mafham M, Bell JL, Linsell L, Staplin N, Brightling C, Ustianowski A, Elmahi E, Prudon B, Green C, Felton T, Chadwick D, Rege K, Fegan C, Chappell LC, Faust SN, Jaki T, Jeffery K, Montgomery A, Rowan K, Juszczak E, Baillie JK, Haynes R, Landray MJ (2021) Dexamethasone in hospitalized patients with covid-19. N Engl J Med 384(8):693-704. https://doi.org/10.1056/ NEJMoa2021436

Sen M, Lahane S, Lahane TP, Parekh R, Honavar SG (2021) Mucor in a viral land: a tale of two pathogens. Indian J Ophthalmol 69:244-252

Serris A, Danion F, Lanternier F (2019) Disease Entities in Mucormycosis. J Fungi 5:23

Tal S, Spectre G, Kornowski R, Perl L (2020) Venous thromboembolism complicated with COVID-19: what do we know so far? Acta Haematol 143:417-424

van Arkel ALE, Rijpstra TA, Belderbos HNA, van Wijngaarden P, Verweij PE, Bentvelsen RG (2020) COVID-19 associated pulmonary aspergillosis. Am J Respir Crit Care Med 202:132-135

Wang D, Hu B, Hu C, Zhu F, Liu X, Zhang J, Wang B, Xiang H, Cheng Z, Xiong Y, Zhao Y, Li Y, Wang X, Peng Z (2020a) Clinical characteristics of 138 hospitalized patients with 2019 novel coronavirusinfected pneumonia in Wuhan, China. JAMA 323:1061-1069

Wang Z, Yang B, Li Q, Wen L, Zhang R (2020b) Clinical features of 69 cases with coronavirus disease 2019 in Wuhan, China. Clin Infect Dis 71: 769-777. [Epubaheadofprint]. https://doi.org/10. 1093/cid/ciaa272

Werthman-Ehrenreich A (2020) Mucormycosis with orbital compartment syndrome in a patient with COVID-19. Am J Emerg Med 42: 264.e5-264.e8. https://doi.org/10.1016/j.ajem.2020.09.032

Yang X, Yu Y, Xu J, Shu H, Xia J, Liu H, Wu Y, Zhang L, Yu Z, Fang M, Yu T, Wang Y, Pan S, Zou X, Yuan S, Shang Y (2020) Clinical course and outcomes of critically ill patients with SARS-CoV-2 pneumonia in Wuhan, China: a single-centered, retrospective, observational study. Lancet Respir Med 8:475-481

Publisher's Note Springer Nature remains neutral with regard to jurisdictional claims in published maps and institutional affiliations. 